Check for updates

Cite this: RSC Adv., 2019, 9, 6267

Received 21st December 2018

Accepted 5th February 2019

DOI: 10.1039/c8ra10491c

rsc.li/rsc-advances

\section{The carbonization of polyacrylonitrile-derived electrospun carbon nanofibers studied by in situ transmission electron microscopy $\dagger$}

\author{
Roland Schierholz, (D) *a Daniel Kröger, ${ }^{a}$ Henning Weinrich, (D) ab Markus Gehring, (D) ab \\ Hermann Tempel, (D) ${ }^{a}$ Hans Kungl, (iD) a Joachim Mayer (D) cde \\ and Rüdiger-A. Eichel (DDbc
}

\begin{abstract}
Cathode structures derived from carbonized electrospun polyacrylonitrile (PAN) nanofibers are a current line of development for improvement of gas diffusion electrodes for metal-air batteries and fuel cells. Diameter, surface morphology, carbon structure and chemical composition of the carbon based fibers play a crucial role for the functionality of the resulting cathodes, especially with respect to oxygen adsorption properties, electrolyte wetting and electronic conductivity. These functionalities of the carbon fibers are strongly influenced by the carbonization process. Hitherto, fibers were mostly characterized by ex situ methods, which require great effort for statistical analysis in the case of microscopy. Here, we show the morphological and structural evolution of nanofibers during their carbonization at up to $1000^{\circ} \mathrm{C}$ by in situ transmission electron microscopy (TEM). Changes in fiber diameter and surface morphology of individual nanofibers were observed at $250{ }^{\circ} \mathrm{C}, 600{ }^{\circ} \mathrm{C}, 800{ }^{\circ} \mathrm{C}$ and $1000{ }^{\circ} \mathrm{C}$ in imaging mode. The structural evolution was studied by concomitant high resolution TEM and electron diffraction. The results show with comparatively little effort shrinkage of the nanofiber diameter, roughening of the surface morphology and formation of turbostratic carbon with increasing carbonization temperature at identical locations.
\end{abstract}

\section{Introduction}

Electrospinning is an efficient technique to provide 1-D nanostructured polymeric or polymer-based materials and composites. ${ }^{1}$ Recent research identified carbonized polymer-derived fibers as a promising class of materials for a wide scope of energy applications, such as in catalyst supports for direct methanol fuel cells, methanol oxidation and water splitting. ${ }^{2-4}$ The catalytic activity for the oxygen reduction reaction in alkaline media makes nitrogen doped carbon materials a promising candidate for cathode structures of metal air batteries. ${ }^{5}$ In addition polyacrylonitrile (PAN)-derived carbon fibers also find application in supercapacitors and as anode structures in lithium-ion batteries. ${ }^{6-8}$ The tunability of the fiber properties relevant for their functionality - wettability, porosity of fiber

${ }^{a}$ Forschungszentrum Jülich $\mathrm{GmbH}$, Institute of Energy and Climate Research, Fundamental Electrochemistry (IEK-9), Germany.E-mail: r.schierholz@fz-juelich.de

${ }^{b}$ Institute of Physical Chemistry, Rheinisch Westfälische Technische Hochschule Aachen, Germany

'Jülich Aachen Research Alliance: JARA-Energy, Germany

${ }^{d}$ Forschungszentrum Jülich GmbH, Ernst Ruska-Centre (ER-C) for Microscopy and Spectroscopy with Electrons, Germany

${ }^{e}$ Central Facility for Electron Microscopy, Rheinisch Westfälische Technische Hochschule Aachen, Germany

$\dagger$ Electronic supplementary information (ESI) available. See DOI: 10.1039/c8ra10491c surfaces, chemical composition and structure of the carbonized fibers - underline the potential of this type of material. ${ }^{9}$ However, a precondition for the tailoring of properties is knowledge and control over the effects of process specifications during the preparation of the polymer solutions, the electrospinning process as well as the crosslinking and the carbonization steps.

After electrospinning the standard procedure to convert polymeric PAN-fibers into carbon fibers is oxidative stabilization, carbonization and graphitization. ${ }^{10}$ The first of these three steps is performed under air between $200{ }^{\circ} \mathrm{C}$ and $300{ }^{\circ} \mathrm{C}$ and the reactions involved are the cyclisation of nitrile and incorporation of oxygen as described by Goodhew et al. ${ }^{11}$ This step is important to avoid votalilization, maximize the carbon yield and avoid the formation of hollow core fibers in the subsequent carbonization step. ${ }^{\mathbf{1 0 , 1 2}}$ The subsequent carbonization step is conducted under inert gas atmosphere up to temperatures of about $1500{ }^{\circ} \mathrm{C}$. For higher temperatures, generally above $2500{ }^{\circ} \mathrm{C}$, the term graphitization is used. ${ }^{\mathbf{1 0}}$ Graphitization is usually performed for "high modulus" carbon fibers, used for reinforced plastics and not discussed in this manuscript, which focuses on carbonization below $1500{ }^{\circ} \mathrm{C}$. The carbonization step involves losses of oxygen and nitrogen, still present after crosslinking. The corresponding evaporation processes start at temperatures just above the stabilization temperature of e.g. $400{ }^{\circ} \mathrm{C}^{13}$ However, most changes were reported for 
temperatures between $700{ }^{\circ} \mathrm{C}$ and $1200{ }^{\circ} \mathrm{C} .{ }^{12}$ Consequently changes in chemistry and structure of the fibers, but also in their dimensions and surface morphology result from the carbonization step in this temperature range.

A wide scope of analytical techniques such as X-ray Photoelectron Spectroscopy (XPS), X-Ray Diffractometry (XRD), Raman, infrared-spectroscopy, thermogravimetry (TGA) and differential thermoanalysis (DTA) has been applied for the characterization of structure, crosslinking/stabilization and carbonization behavior of PAN-derived carbon fibers., 14-20 While most of these methods provide an overall view on the fiber materials, a technique of choice in order to obtain localized information - in particular on the fiber surfaces - is transmission electron microscopy (TEM).

Most TEM-studies were performed to study strength structure-relationship of carbon microfibers. In 1976 for example Bennett ${ }^{21}$ did an extensive three-dimensional analysis of PAN-derived carbon fibers heat treated at $1000{ }^{\circ} \mathrm{C}, 1500{ }^{\circ} \mathrm{C}$ and $2500{ }^{\circ} \mathrm{C}$ using various TEM techniques such as bright and dark field TEM, high resolution transmission electron microscopy (HRTEM) and quantitative electron diffraction analysis on transverse and longitudinal cross-sections. A more recent HRTEM investigation of copolymerized acrylonitrile/itaconic acid fibers drawn from a spinneret in a coagulation bath is presented by Bai et al. ${ }^{22}$ Here, amorphous and ordered structures were identified even without heat treatment. Furthermore, while highly oriented structures were detected in the longitudinal sections, the cross-sections showed onion like spherical ordering as well as crystallites.

Laffont et al. performed HRTEM, electron energy loss spectroscopy (EELS) and XRD on different types of commercial PANderived carbonized microfibers. They report coherent turbostratic graphite with a $d$-spacing larger than $3.43 \AA^{23}$ This is larger than the value of $3.35 \AA$ published for graphite. ${ }^{24,25}$ Moreover, the size of the stacks firstly remains small with $L_{10} \leq$ $4 \mathrm{~nm}$ (parallel to the graphitic planes) and $L_{002} \leq 1.3 \mathrm{~nm}$ (perpendicular to the graphitic planes), but increases with processing temperature from $300{ }^{\circ} \mathrm{C}$ to $1000{ }^{\circ} \mathrm{C} .{ }^{23}$ EELS analysis of the K-edges of carbon, nitrogen and oxygen shows that a carbon content of $99 \%$ is achieved for carbonization at $1000{ }^{\circ} \mathrm{C}$, while only little amount of oxygen $(0.5-1 \%$ from initially $1-4 \%$ ) remains in the fibers. ${ }^{23}$ More dramatic is $I$ the change of nitrogen content from initially $1-12 \%$ it drops to zero after $1000{ }^{\circ} \mathrm{C}$, after $800{ }^{\circ} \mathrm{C} 1-3 \%$ are reported. ${ }^{23}$ In addition Laffont et al. also studied the $\sigma+\pi$ plasmon in the low loss region and show that the $\sigma$-plasmon shifts to higher energies for materials processed at higher temperature and correlate this with lower resistivity. ${ }^{23}$ In a later work Laffont et al. used a combination of EELS and XPS to study the bonding situation especially of nitrogen. ${ }^{26}$ In this study, they report an increase of about +7 at $\%$ during stabilization in air at $250{ }^{\circ} \mathrm{C}$ and a loss of up to -15 at\% during subsequent carbonization at up to $1000{ }^{\circ} \mathrm{C}$ in $\mathrm{N}_{2}$-atmosphere. Depending on the fiber treatment after spinning significant amounts of nitrogen $[\mathrm{N}] /[\mathrm{C}] \approx 0.1$ and oxygen $[\mathrm{O}] /[\mathrm{C}] \approx 0.05$ remain in the fiber. The chemical composition and bonding situation of nitrogen in carbon fibers were also studied in detail by other groups using XPS to distinguish different bonding types of nitrogen for application in batteries and fuel cells., ${ }^{\mathbf{2 1 4 , 1 9}}$

The previously described results were mostly related to microfibers with diameters ranging from 5 to $12 \mu \mathrm{m}$. Musiol et al. compared PAN-derived nano and microfibers heat treated at $1000{ }^{\circ} \mathrm{C}, 2000{ }^{\circ} \mathrm{C}$ and $2800{ }^{\circ} \mathrm{C}$, and reported higher massloss for nanofibers, $61 \%$ residual mass for carbon microfibers and $45 \%$ residual mass for carbon nanofibers after a heat treatment at $1000{ }^{\circ} \mathrm{C} .{ }^{18}$ HRTEM and Raman spectroscopy showed graphitization for fibers treated above $2000{ }^{\circ} \mathrm{C}$ while the $1000{ }^{\circ} \mathrm{C}$ fibers still appear quite amorphous. ${ }^{18}$ For the temperature range from $1500{ }^{\circ} \mathrm{C}$ to $2800{ }^{\circ} \mathrm{C}$ a development from relatively smooth to rough and ridged morphology was reported by Kurban et al. ${ }^{27}$ In all reports temperature ranges differ and also a variety of different investigation techniques is applied. It is shown that the change of chemical composition; carbon structure and morphology of the fibers strongly depend on the carbonization process as well as the treatment applied in the previous steps. $^{18,26,27}$

Common feature of most microscopy investigations is that the comparison of structural and morphological characteristics was performed based on ex situ experiments. Indeed, ex situ analyses are advantageous with respect to flexibility of the fibers processing conditions as they are not limited by the in situ experiment. However, comparisons of fibers subject to small process variations require that the effects of the process variation exceed the scattering in characteristics of individual fibers. Otherwise, large efforts by statistical analysis of many samples are required to provide significant data for establishing correlations between processing parameters and structural as well as microstructural characteristics. An approach to circumvent this is, to investigate the evolution of fiber characteristics on identical locations on individual fibers during processing with in situ microscopy methods. A first attempt was applied by Prilutsky et al. for the case of the carbonization of electrospun PAN nanofibers containing carbon nanotubes in $2010 .{ }^{28}$ However, in their study a heating stage, where the whole $3 \mathrm{~mm}$ grid is heated up, was used. Due to their larger heating volume such heating stages as a result feature slow response times, inaccurate temperatures, sample instabilities and strong thermal drift, thus limit the TEM image resolution. In this study we aim on studying the shrinkage of fiber diameter, surface morphology as well as structural changes on the same fibers in one single in situ TEM heating experiment at four subsequent temperature stages $-250{ }^{\circ} \mathrm{C}$, after oxidative stabilization, $600{ }^{\circ} \mathrm{C}, 800{ }^{\circ} \mathrm{C}$ and $1000{ }^{\circ} \mathrm{C}$ on an in situ heating holder based on micromechanical systems (MEMS). ${ }^{29}$ In contrast to the before mentioned system this in situ holder allows high resolution TEM images and electron diffraction in accurate temperatures without instabilities, which will help to acquire a conclusive picture for the evolution of electrospun PAN nanofibers during carbonization.

\section{Experimental}

\section{Materials preparation}

PAN nanofibers were prepared from a dimethylformamide (DMF) solution $>99 \%$ (Sigma Aldrich) containing $10 \mathrm{wt} \%$ PAN 
(molecular weight 150 000, Sigma Aldrich) using an IME EC-CLI (IME, Netherlands) electrospinner. The device was setup with a $0.8 \mathrm{~mm}$ nozzle diameter, a rotating cylindrical target (diameter $=20 \mathrm{~mm}$ ) and a nozzle-to-target distance of $160 \mathrm{~mm}$. An electric field of $15 \mathrm{kV}$ was applied between nozzle and collector in a processing chamber, which was kept at $25{ }^{\circ} \mathrm{C}$ and $20 \%$ relative humidity. The nozzle and the target were operated at lateral movement of $20 \mathrm{~mm} \mathrm{~s}^{-1}$ within a range of $100 \mathrm{~mm}$ and a rotation speed of $1500 \mathrm{rpm}$ respectively. The feed rate for the

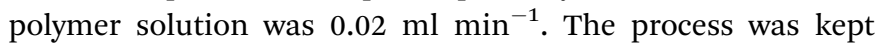
running for $30 \mathrm{~min}$ resulting in polymeric nanofiber mats of $100 \mathrm{mg}$ with dimensions of $60 \times 100 \times 0.05 \mathrm{~mm}$ approximately. The polymeric nanofiber mats were dried in a cabinet at $200{ }^{\circ} \mathrm{C}$ overnight to evaporate the remaining solvent. Oxidative stabilization and partial cross-linking was performed in air at $250{ }^{\circ} \mathrm{C}$ for $4 \mathrm{~h}$.

\section{SEM characterization}

Fiber mats were characterized by SEM (FEI, Quanta FEG 650) using an acceleration voltage of $2 \mathrm{kV}$. A micrograph of an oxidatively stabilized, non-carbonized fiber mat is shown in Fig. S1.† Fiber diameters were measured manually with Olympus Stream Essentials Desktop 1.9.3.

\section{TEM sample preparation}

A dispersion of nanofibers in their oxidative stabilized state, after the crosslinking step at $250{ }^{\circ} \mathrm{C}$, was prepared by ultrasonic treatment of a piece of the nanofiber mat in pure ethanol. A droplet of the dispersion was applied to the heating chip of a MEMS based in situ heating holder (DENSsolutions) with
$\mathrm{Si}_{3} \mathrm{~N}_{4}$ membrane and carbon coated windows. By means of focused ion beam holes of circular shape were previously etched into the carbon film to provide areas without carbon support for the experiment. ${ }^{30}$

\section{In situ carbonization conditions}

The temperature - time profile applied for the carbonization process during the in situ experiment is depicted in Fig. 1. The profile is designed for nanofiber characterization during four subsequent temperature stages $-250{ }^{\circ} \mathrm{C}, 600{ }^{\circ} \mathrm{C}, 800{ }^{\circ} \mathrm{C}$ and $1000{ }^{\circ} \mathrm{C}$. All fibers located on the heating chip were heated simultaneously. Heating rate applied during all temperature steps was $15{ }^{\circ} \mathrm{C} \min ^{-1}$. Dwell times at the individual temperature steps were varying between 5 and $7 \mathrm{~h}$ according to the time required for TEM image acquisition of all nanofibers. In total, the in situ experiment was carried out on six fibers with three observations during each of the four different temperature steps. The temperature steps were split into several days of microscope time.

For three fibers (1, 3 and 6$)$ investigations were limited to low resolution size analysis, which is supposed to involve only minor influence of the electron beam. Three other fibers $(2,4$ and 5) were subject to high resolution imaging and diffraction, which implies substantially higher dose for the high resolution and longer exposure to the beam during the alignment of the microscope for electron diffraction. The atmosphere during the in situ carbonization was imposed by the ultra-high vacuum (UHV) conditions required in the TEM. ${ }^{31}$ After every heating period the sample holder was cooled down to ambient temperature by switching off the heating.

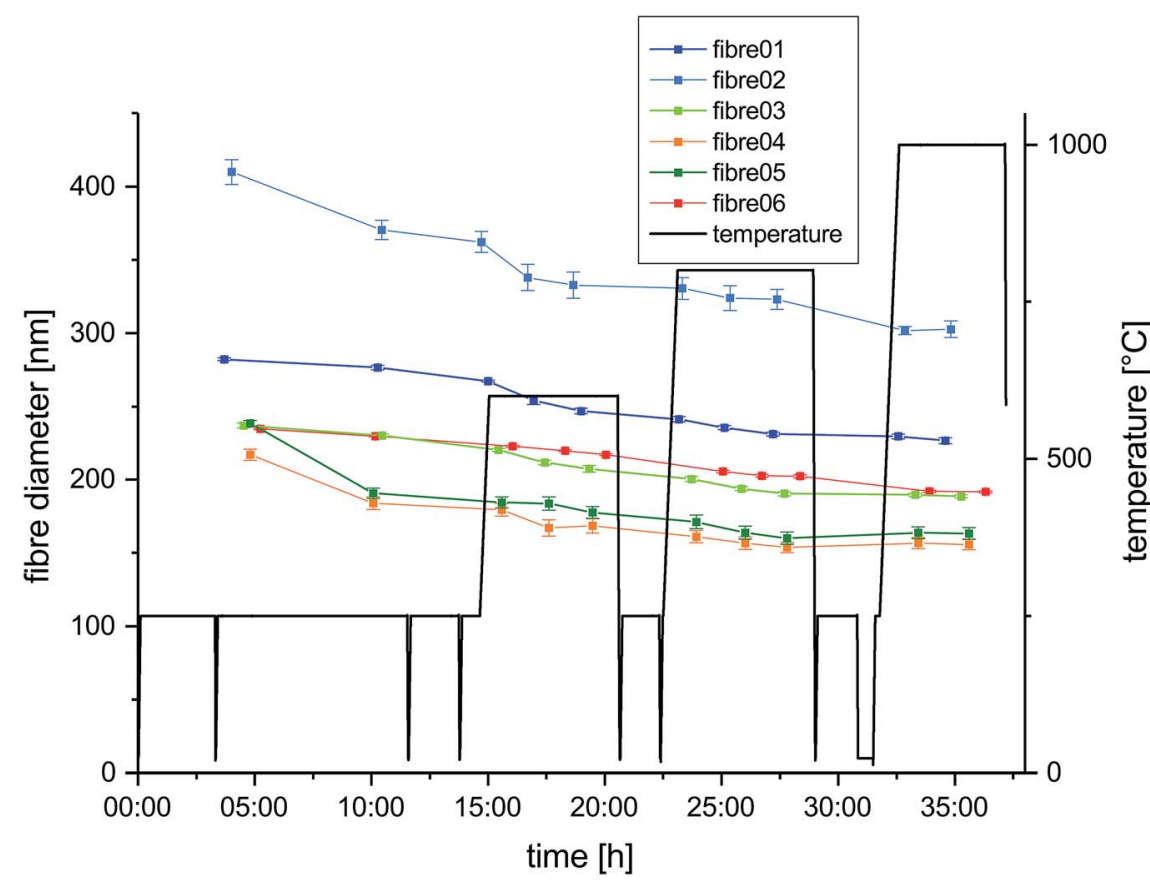

Fig. 1 Heating program applied during experiment (black) and the measured diameters at different stages of the experiments for six selected nanofibers are plotted as a function of time (colored). 


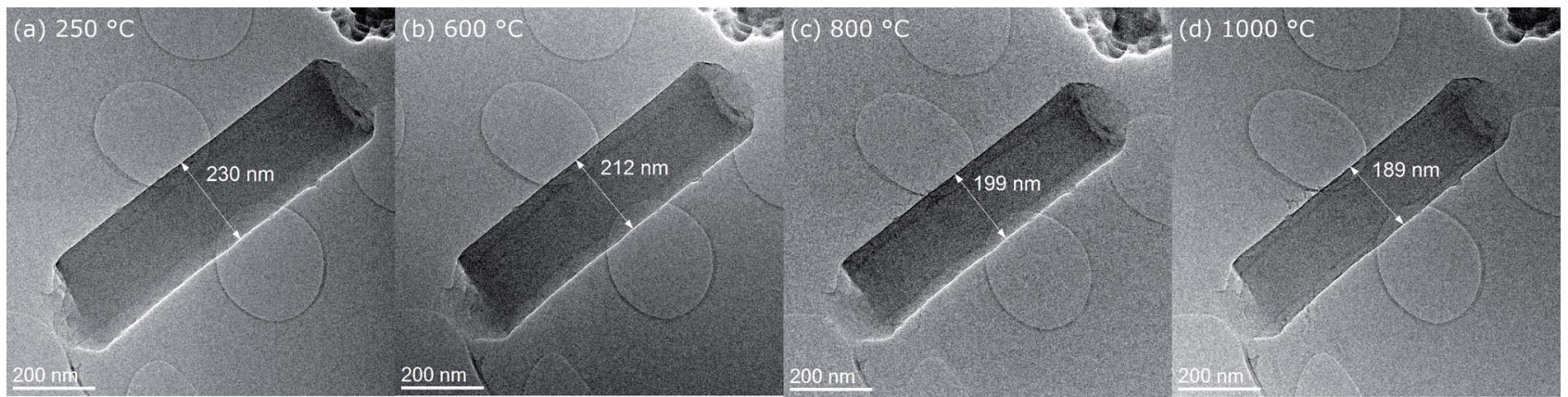

Fig. 2 Overview images of fiber 6 at different stages during the in situ experiment (a) $250{ }^{\circ} \mathrm{C}$, (b) $600{ }^{\circ} \mathrm{C},(\mathrm{c}) 800^{\circ} \mathrm{C}$ and (d) $1000^{\circ} \mathrm{C}$.

\section{TEM experiments}

In situ TEM experiments were carried out on a FEI Titan with a spherical aberration $\left(C_{\mathrm{s}}\right)$ corrector (CEOS) for the objective lens and operated at $300 \mathrm{kV}$ using the negative- $C_{\mathrm{s}}$ imaging technique, which provides images with high contrast and low noise. ${ }^{31,32}$ In imaging mode the evolution of fiber diameters, fiber morphologies and alignment of the graphite layers was analyzed by low, intermediate and high resolution images respectively. The high resolution images were taken from lateral and fracture surfaces of the nanofibers. Diffraction patterns were taken from one nanofiber at a camera length of $L=$ $490 \mathrm{~mm}$ where the $\{002\}$ carbon reflection is not covered by the beam stop and the second and third diffraction rings are still visible. Images with the same settings were taken two to three times every two hours during each temperature step.

\section{Results}

\section{Fiber diameter}

Fiber diameters were measured on six different nanofibers at different stages in course of the in situ experiment and the evolution of their diameters over time is plotted together with the temperature profile in Fig. 1. All selected nanofibers have initial diameters in a range from $200 \mathrm{~nm}$ to just above $400 \mathrm{~nm}$, which is typical for the processing used and in agreement with the fiber diameter distribution measured from SEM micrographs of the fiber mats (Fig. $\mathrm{S} 1$ and $\mathrm{S} 2 \dagger$ ). The evolution of the diameter of fiber 6 along with the subsequent temperature treatments at $250{ }^{\circ} \mathrm{C}, 600{ }^{\circ} \mathrm{C}, 800{ }^{\circ} \mathrm{C}$ and $1000{ }^{\circ} \mathrm{C}$ is shown exemplary in low magnification TEM images in Fig. 2(a-d). The shrinkage behavior is representative for a nanofiber carbonized under the influence of temperature and UHV, but largely unaffected by the electron beam. Very similar changes in diameters are observed for fiber 1 and 3, with minimized exposure to the electron beam. The decrease in diameter over the complete cycle is in the range between 15 and $20 \%$ compared to the initial diameter. In contrast to that, the three nanofibers exposed to the influence of the electron beam for extended time spans (fibers 2, 4 and 5) show larger shrinkage in diameter mounting up to $32 \%$. This behavior is independent of the initial nanofiber diameters (Fig. 1). The enhanced shrinkage under the influence of the electron beam occurred in particular during the $250{ }^{\circ} \mathrm{C}$ annealing step in the microscope. At higher temperature, no significant differences between the shrinkage rates can be identified.

\section{Nanofiber morphology}

The surface morphology can be studied at intermediate magnification micrographs as shown for fiber 5 in Fig. 3(a-d). In the initial state at $250{ }^{\circ} \mathrm{C}$ in Fig. 3(a), the stabilized nanofiber appears smooth with homogeneous amorphous contrast. At $600{ }^{\circ} \mathrm{C}$, the roughness markedly increased (Fig. 3(b)) and it appears like nanosized particles are sticking out from the fiber. Also diffraction contrast arises throughout the nanofiber with brighter and darker regions with similar sizes around $5 \mathrm{~nm}$. With further increase of temperature to $800^{\circ} \mathrm{C}$ and $1000{ }^{\circ} \mathrm{C}$ the

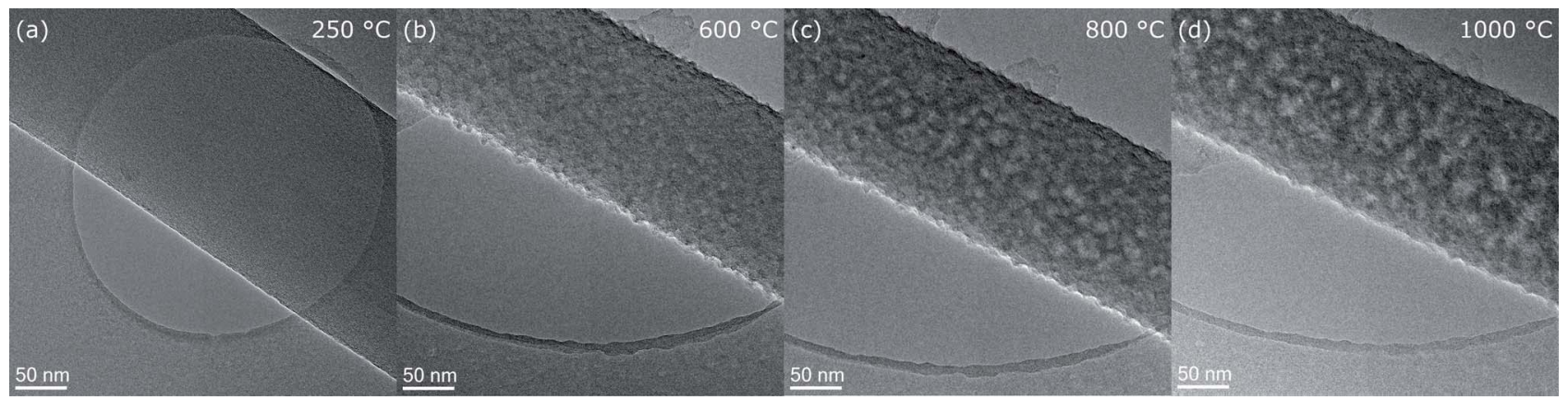

Fig. 3 Intermediate resolution images showing the development of fiber 5 morphology at temperature steps (a) $250{ }^{\circ} \mathrm{C}$, (b) $600{ }^{\circ} \mathrm{C}$, (c) $800{ }^{\circ} \mathrm{C}$ and (d) $1000^{\circ} \mathrm{C}$. 
lateral size of the objects producing the roughness and the diffraction contrast, most probably turbostratic carbon, increases further to $\approx 10 \mathrm{~nm}$ at $800{ }^{\circ} \mathrm{C}$ and even more up to $15 \mathrm{~nm}$ at $1000{ }^{\circ} \mathrm{C}$. However, the roughness itself does not continue to increase significantly between 600 and $1000{ }^{\circ} \mathrm{C}$.

\section{Carbon structure analysis by HRTEM}

To evaluate the morphological changes of the nanofiber surface and the atomic structure in more detail HRTEM micrographs of the lateral surface of fiber 5 at $250{ }^{\circ} \mathrm{C}, 600{ }^{\circ} \mathrm{C}, 800{ }^{\circ} \mathrm{C}$ and $1000{ }^{\circ} \mathrm{C}$ are shown in Fig. 4(a-d). Furthermore, Fig. 4(e-h) shows HRTEM micrographs of the tip of fiber 4 . For the initial state of the stabilized PAN-nanofiber at $250{ }^{\circ} \mathrm{C}$ (Fig. 4(a) and (e)) the HRTEM micrographs show an amorphous contrast and also the fast Fourier transforms (FFTs), shown in the insets, display only diffuse rings. At $600{ }^{\circ} \mathrm{C}$ (Fig. 4(b) and (f)), the ordering of the carbon atoms, mainly (002)-planes with $d_{002} \approx 0.35 \mathrm{~nm}$, becomes visible in real space. At the lateral surface of the fiber, this ordering appears preferentially parallel to the fiber axis leading to higher intensity in the diffractogram perpendicular to the fiber axis. At $600{ }^{\circ} \mathrm{C}$, only few planes are stacked, which corresponds to $L_{002}$ in the range of few $\mathrm{nm} .{ }^{23}$ Also the lateral size corresponding to $L_{10}$ of these turbostratic regions is in the range of few nm. ${ }^{23}$ At $800{ }^{\circ} \mathrm{C}$, lateral and stacking size is around $5 \mathrm{~nm}$. The number of planes stacked and their lateral size increases markedly in the last heating step from $800{ }^{\circ} \mathrm{C}$ (Fig. 4(c) and (g)) to $1000{ }^{\circ} \mathrm{C}$ (Fig. 4(d) and (h)). Here, turbostratic regions become as large as $10 \mathrm{~nm}$, in agreement with the diffraction contrast observed in the intermediate resolution images in Fig. 3(c and d). This evolution can also be followed in the FFTs of the images shown in the insets. In the initial state at $250{ }^{\circ} \mathrm{C}$ (Fig. 4(a) and (e)), only two very diffuse rings are present. At $600{ }^{\circ} \mathrm{C}$ (Fig. 4(b) and (f)), these rings get more defined and especially for the 002ring a texture appears for the HRTEM images recorded at the side of fiber 5 (Fig. 4(b)). The 002-ring shows markedly higher intensity perpendicular to the fiber surface proving the preferential alignment of (002)-planes parallel to the surface. At $600{ }^{\circ} \mathrm{C}$ (Fig. 4(b) and (f)) and $800{ }^{\circ} \mathrm{C}$ (Fig. 4(c) and (g)), the former second diffuse ring splits into two rings. At $1000{ }^{\circ} \mathrm{C}$ (Fig. 4(d) and (h)), these two rings are clearly distinguishable. In the FFTs of the images recorded at the lateral surface of the nanofiber, the inner of these two rings with smaller scattering vectors shows high intensity parallel to the fiber axis and the outer ring has the maximum intensity perpendicular to the fiber axis like the 002-ring. Comparing the scattering vectors and relative intensities for reflections of graphite listed in Table $\mathrm{S} 1, \dagger$ the first subring of the second ring can be attributed to $\{100\}$ - and $\{101\}$ planes, which are perpendicular to $\{002\}$-planes or form an angle of $\approx 72^{\circ}$ with them (see also Fig. 5(a)).

The $\{102\}-,\{004\}$ - and $\{103\}$-planes contribute to the second subring with $g_{h k l}$ in the range from 5.5 to $6.5 \mathrm{~nm}^{-1}$. Due to their multiplicity and orientation the $\{102\}$ - and $\{103\}$-planes form a rather homogenously distributed intensity in the azimuthal range. The texture observed within the second subring arises mainly from 004, the second order reflection of the (002)-planes. In the proposed structure sketched in Fig. 6(a), close to the nanofiber surface most of the graphitic (002)-planes are parallel to the electron beam and in diffraction condition. However, the rotation around the $c$-axis remains a degree of freedom and therefore the relative intensity of 002 and 004 is expected to be higher, compared to $h 0 l$ - and $h k l$-reflections, which are excited

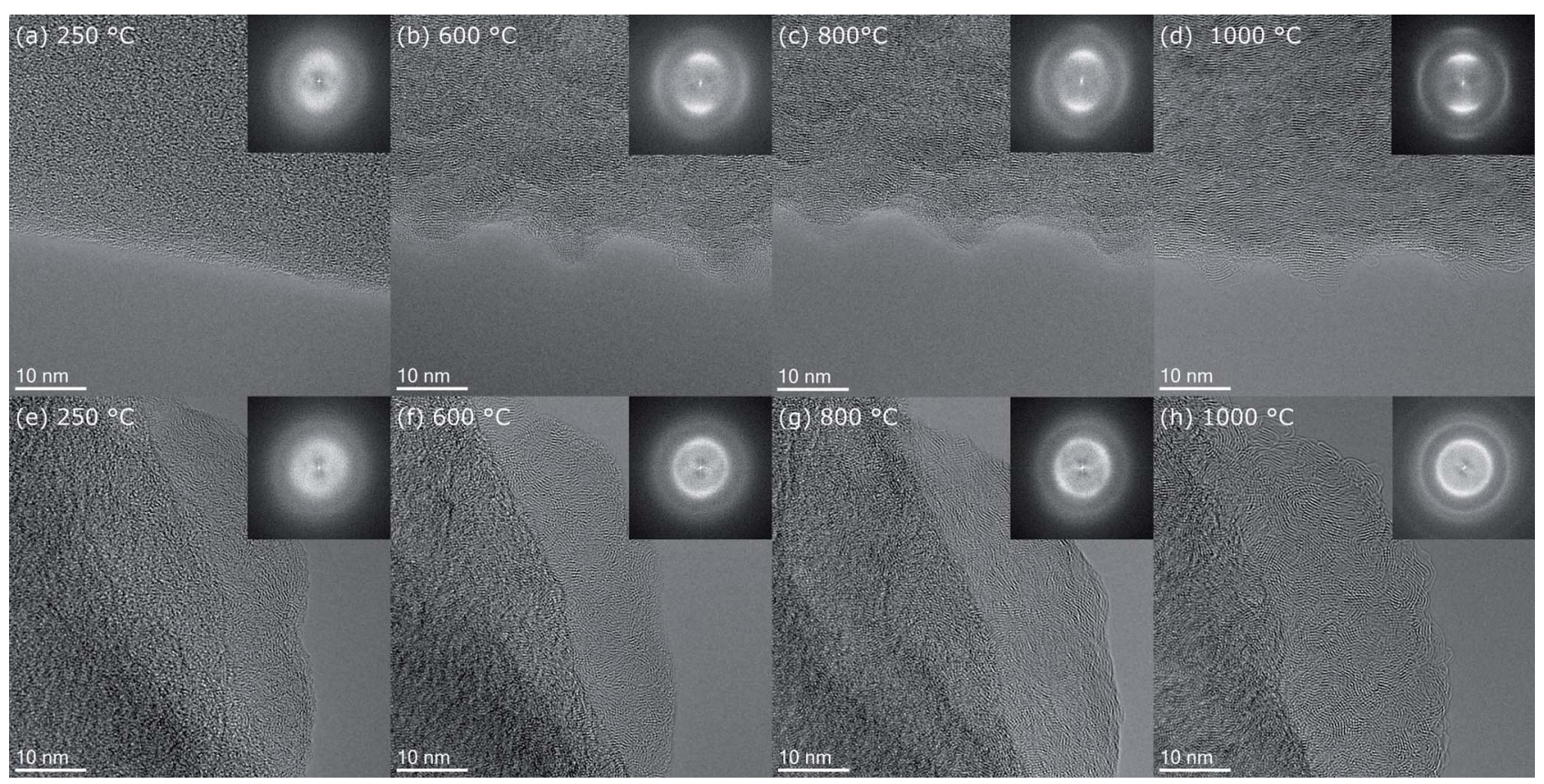

Fig. 4 HRTEM images of the side surface of fiber 5 at temperature steps (a) $250{ }^{\circ} \mathrm{C}$, (b) $600{ }^{\circ} \mathrm{C}$, (c) $800^{\circ} \mathrm{C}$ and (d) $1000^{\circ} \mathrm{C}$. FFT's are shown on the insets. HRTEM images and corresponding FFT's of a thin part at the tip of fiber 4 at the four temperatures (e) $250{ }^{\circ} \mathrm{C},(\mathrm{f}) 600^{\circ} \mathrm{C},(\mathrm{g}) 800^{\circ} \mathrm{C}$ and (h) $1000^{\circ} \mathrm{C}$ 
(a)

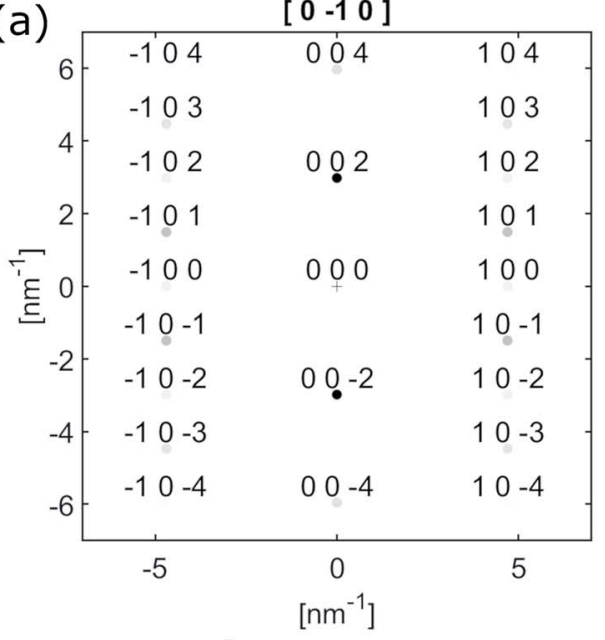

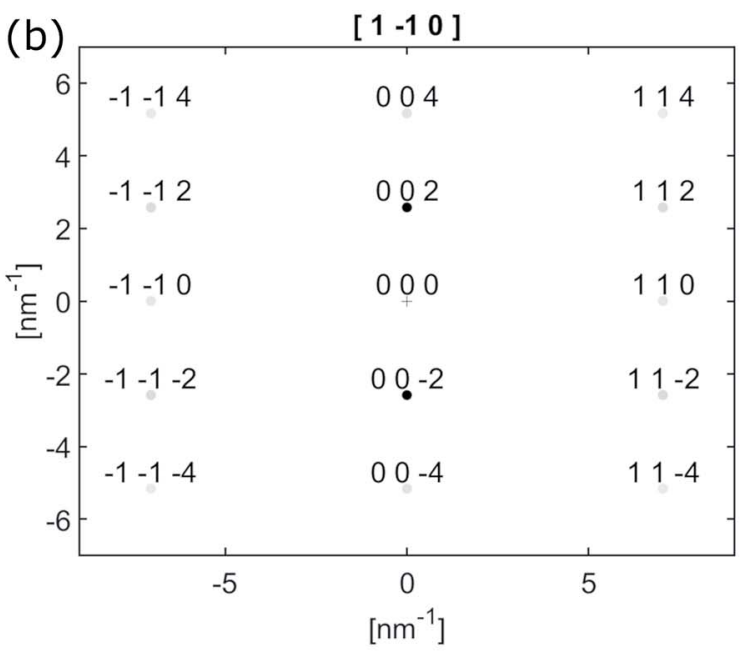

(c)

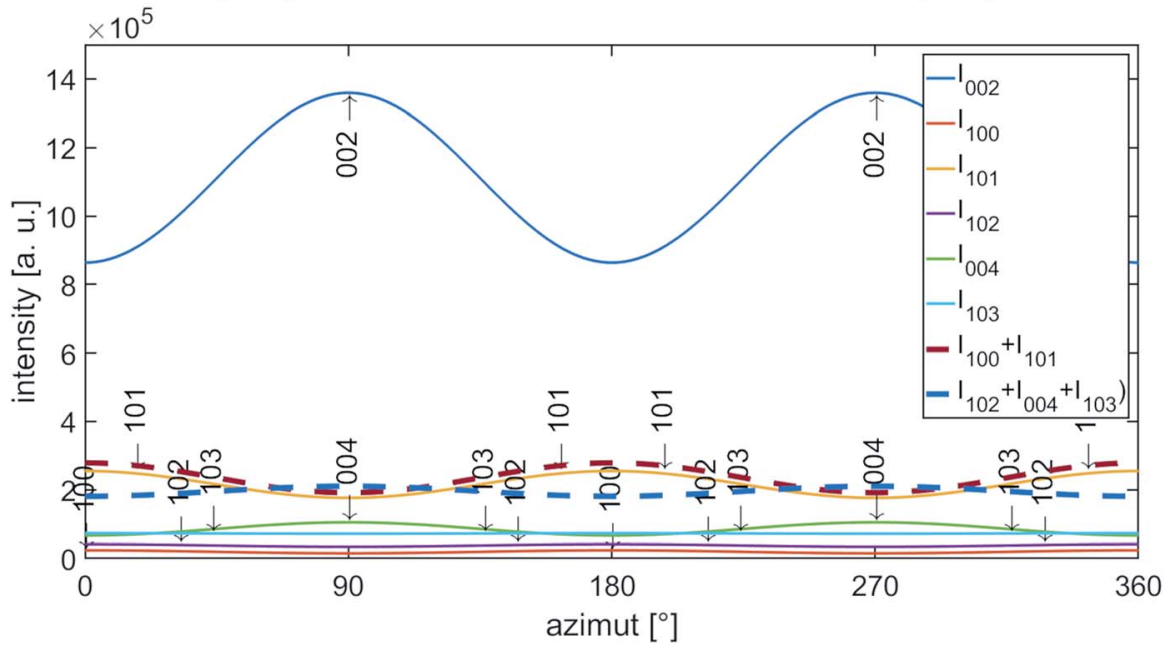

Fig. 5 (a) [0-10] and (b) [1-10] electron diffraction pattern of single crystalline graphite. (c) Calculated azimuthal intensities for reflection up to 103 with $\left|g_{h k l}\right| \leq 6.5 \mathrm{~nm}^{-1}$.
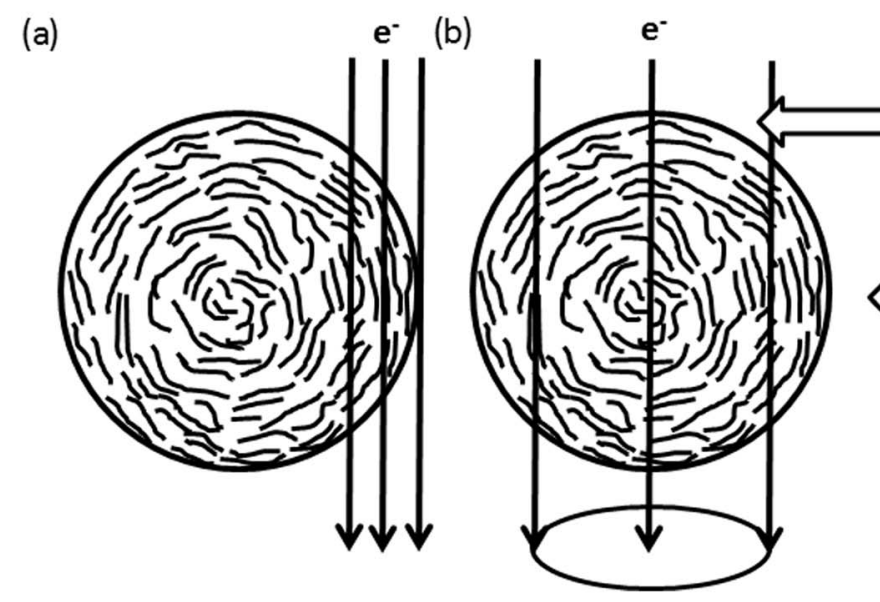

SA

Fig. 6 Schematic sketch of the nanofiber texture showing the preferential alignment of $\{002\}$-planes parallel to the nanofibers surface. (a) Shows conditions of HRTEM images recorded at the side of the nanofiber in Fig. 4(a) - (d). In (b) the condition for Electron Diffraction (ED) with a selected area Aperture (SA) positioned in the central part of the nanofiber, as applied for electron diffraction in Fig. 7, is shown. The rotation around the $c-$ axis remains a degree of freedom within the graphitic planes. 
for graphitic regions in $\langle 100\rangle$ and $\langle 1-10\rangle$ zone axis orientations only, as depicted in Fig. 5(a) and (b).

The observed variation of scattered intensities with the azimuth could be reproduced by calculations using a simple model. Starting with the $[0-10]$ zone axis diffraction pattern as depicted in Fig. 5(a) the variety of c-axis orientation within the plane perpendicular to the electron beam is introduced by a Gaussian distribution with $\sigma=60^{\circ}$ around the azimuthal positions of the reflections. Furthermore the relative intensities of $h 0 l$ - and $h k l$-reflections are artificially decreased by a factor of 0.5 to represent relative intensities of the higher order reflections in respect to 002 . The resulting intensity for reflections up to 103 $\left(g_{h k l} \leq 6.5 \mathrm{~nm}^{-1}\right)$ is plotted as a function of the azimuthal angle in Fig. 5(c). An azimuthal angle of $0^{\circ}$ and $180^{\circ}$ corresponds to scattering parallel to the fiber axis, $90^{\circ}$ and $270^{\circ}$ perpendicular to the fiber axis. The thick dashed lines represent $I_{100}+I_{101}$ (red) and $I_{102}+I_{004}+I_{103}$ (blue), which in first approximation can be attributed to the inner and outer subring of the former diffuse second ring in the FFTs in Fig. 4(b-d). Furthermore, the graph represents the higher intensity parallel to the fiber axis for the inner subring (red) and the higher intensity of the second subring (blue) perpendicular to the fiber axis.

Enhanced ordering in the graphitic structure under the influence of high temperatures is also visible at fracture surfaces at the tip of the nanofiber pieces (Fig. 4(e-h)). In contrast to the turbostratic structures formed on the lateral surfaces no preferential orientation of the turbostratic areas can be recognized in the real space image. Confirming this observation, the FFTs shown in the insets of Fig. 4(e-h) show rings with homogeneously distributed intensity in the azimuthal range as expected for randomly oriented crystallites. With increasing temperature these rings just get sharper and more defined. The interpretation of these observations is sketched in Fig. 6. As in the central part of the nanofiber the distance to the surface is comparable in all directions, so the ordered regions in this part of the nanofiber have arbitrary orientations among each other. Thus we conclude that ordering of carbon atoms in a turbostratic form applies also to the inner parts of the
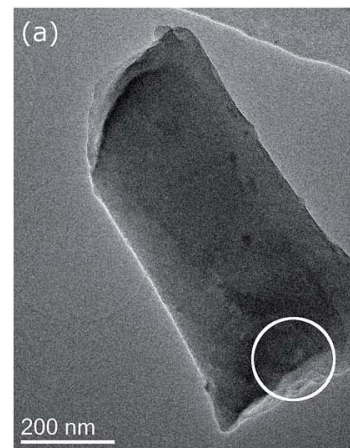

(b)
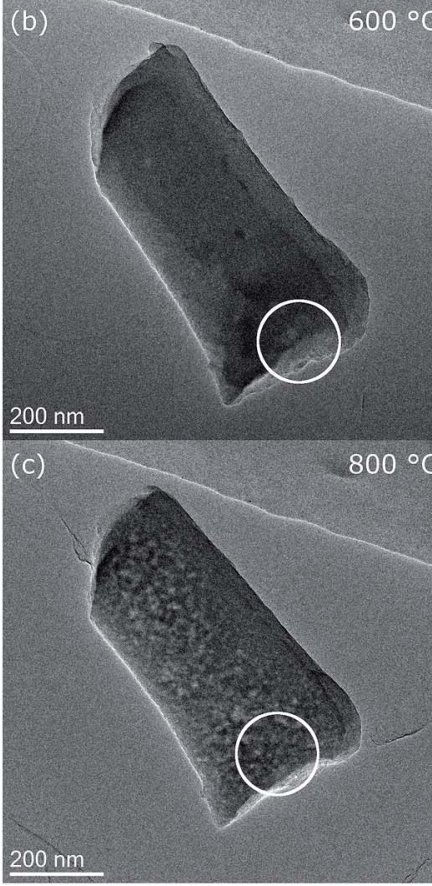

$250^{\circ} \mathrm{C}(\mathrm{d})$
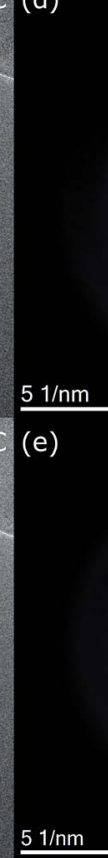

(f)
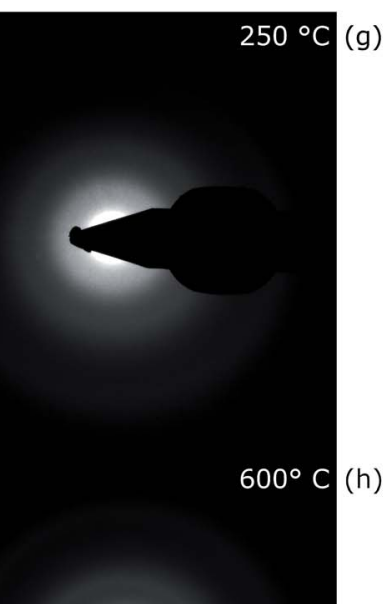

\section{$250{ }^{\circ} \mathrm{C}(\mathrm{g})$}
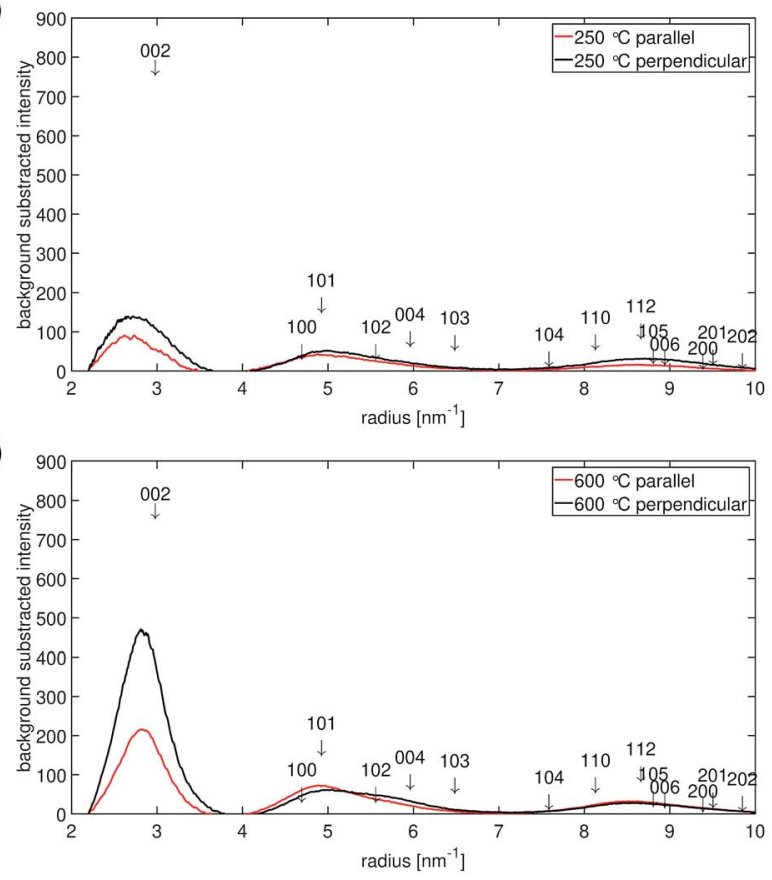

$800{ }^{\circ} \mathrm{C}(\mathrm{i})$

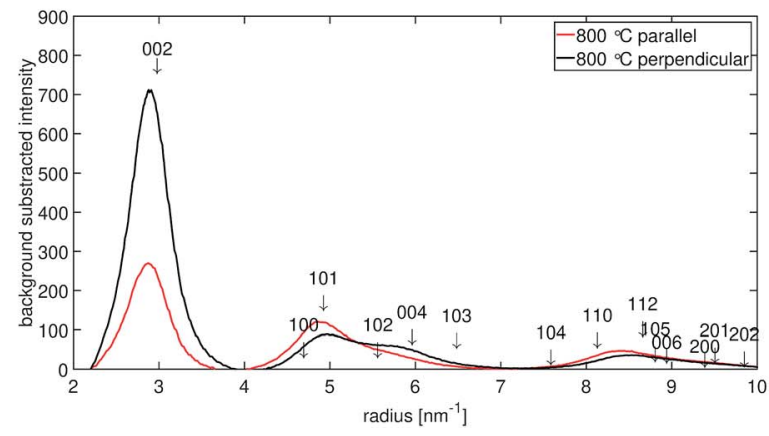

Fig. 7 Fiber 2 selected for electron diffraction experiment at temperature stages (a) $250{ }^{\circ} \mathrm{C}$, (b) $600{ }^{\circ} \mathrm{C}$ and (c) $800{ }^{\circ} \mathrm{C}$. The position of the selected area aperture (SA) is marked by a white circle. ( $d-f)$ Show the corresponding electron diffraction patterns, ( $g$-i) the background subtracted radial intensity parallel and perpendicular to the fiber axis at the corresponding temperatures. 
nanofiber. The 002-texture on the other hand is predominantly formed close to the nanofiber surface.

\section{Carbon structure from electron diffraction}

Fig. 7 shows micrographs of fiber 2 selected for electron diffraction in $(\mathrm{a}-\mathrm{c})$ and the corresponding diffraction patterns recorded at $250{ }^{\circ} \mathrm{C}, 600{ }^{\circ} \mathrm{C}$ and $800{ }^{\circ} \mathrm{C}$ in $(\mathrm{d}-\mathrm{f})$. The position of the selected area aperture (SA) with a size of $\approx 170 \mathrm{~nm}$ in the intermediate image plane is displayed by a white circle. In contrast to HRTEM, in this experiment the complete thickness of the nanofiber inside the aperture contributes to the electron diffraction pattern as sketched in Fig. 6(b). As the aperture size does not include the complete nanofiber diameter, the diffraction patterns reveal a bit more the structure of the central part plus the upper and the lower surface of the nanofiber. This is complementary to the FFTs of the high-resolution images obtained at the fiber surface on the side of the nanofiber, which is excluded by the aperture. The areas selected for recording the pattern were chosen close to the broken tip of the nanofiber to contain only small fraction of fracture edge, but also allowing a precise location of the SA at different stages of the in situ experiment in the TEM.

At $250{ }^{\circ} \mathrm{C}$, three diffuse rings can be recognized in the diffraction pattern (Fig. 7(d)). At $600{ }^{\circ} \mathrm{C}$ (Fig. 7(e)), mainly the first, the 002-ring, becomes more defined and already starts to exhibit higher intensity for scattering vectors perpendicular to the fiber axis. For the second ring the intensity increase at low scattering vectors $\approx 4.6 \mathrm{~nm}^{-1}$ becomes sharper while the decay to larger scattering vectors stays rather diffuse. At $800{ }^{\circ} \mathrm{C}$ (Fig. 7(f)), the texture in the 002-ring becomes more pronounced and the former second diffuse ring splits into two distinguishable rings, of which the first one at lower scattering vectors is of higher intensity.The evolution of the diffraction patterns as described above can be followed in the background subtracted radial intensity, which was extracted from the diffraction patterns plotted in Fig. $7(\mathrm{~g}-\mathrm{i})$. An exponential background in the form of eqn (1)

$$
a_{1} \mathrm{e}^{-b_{1}\left(\mu_{1}-x\right)}+a_{2} \mathrm{e}^{-b_{2}\left(\mu_{2}-x\right)}+d
$$

was fitted to the decaying intensity of the primary beam. To accommodate the texture effects we extracted the radial intensity over an azimuthal range of $\pm 20^{\circ}$ parallel and perpendicular to the fiber axis. This corresponds to an azimuthal range from $215^{\circ}$ to $255^{\circ}$ (perpendicular) and from $305^{\circ}$ to $345^{\circ}$ (parallel) in the diffraction patterns in Fig. $7(\mathrm{~d}-\mathrm{f}) .0^{\circ}$ and $360^{\circ}$ represent the

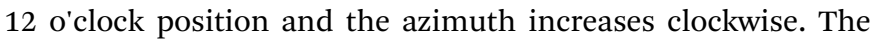
radial intensities are plotted in Fig. $7(\mathrm{~g}-\mathrm{i})$. In all plots arrows indicate the positions of reflections of crystalline graphite and their vertical positions represent the relative intensities of these reflections as listed in Table $\mathbf{S 1 . \dagger}$

Within the diffraction patterns, only the first ring originates from a single reflection, which is 002 . The position of its maximum at $800{ }^{\circ} \mathrm{C}$ is found at $\approx 2.84 \mathrm{~nm}^{-1}$ corresponding to a $d_{002}$-spacing of $352 \mathrm{pm}$. The second ring already contains five reflections, 100, 101, 102, 004 and 103. The sharp increase of intensity at low scattering vectors can be attributed to the rather closely packed $100\left(4.694 \mathrm{~nm}^{-1} I_{\mathrm{rel}} \approx 3 \%\right)$ and $101\left(4.925 \mathrm{~nm}^{-1}\right.$ $I_{\text {rel }} \approx 18 \%$ ). Among the five reflections contributing the second ring 101 is by far the most intense reflection. The diffuse decay to larger scattering vectors can be attributed to more broadly distributed $102\left(5.560 \mathrm{~nm}^{-1} I_{\text {rel }}=3 \%\right), 004\left(5.961 \mathrm{~nm}^{-1} I_{\text {rel }}=\right.$ $7 \%)$ and $103\left(6.482 \mathrm{~nm}^{-1} I_{\text {rel }}=5 \%\right)$ reflections. At $800{ }^{\circ} \mathrm{C}$, two separate rings become distinguishable in the formerly second ring, which can be attributed to these previously described two groups of reflections. We attribute the first subring to 001 and 101, the second subring to 102, 004 and 103 reflections. As the first subring is dominated by 101, it shows higher intensity parallel to the fiber axis. For the second subring the highest intensity is observed for scattering perpendicular to the fiber axis, which can only arise from 004 . Both observations can be explained by the calculated azimuthal intensity distribution shown in Fig. 6(c). The same texture also influences the relative intensity of the reflections under the third main ring in the range from $\approx 7.5 \mathrm{~nm}^{-1}$ to $\approx 10 \mathrm{~nm}^{-1}$. In this range $110(5.4 \%)$ and $112(8.7 \%)$ are the most intense reflections and show higher intensity parallel to the fiber axis.

The texture evolution can be followed by comparing the background subtracted radial intensities parallel and perpendicular to the fiber axis. At $250{ }^{\circ} \mathrm{C}$, no significant difference between scattering parallel and perpendicular (b) to the fiber axis is noticed. At $600{ }^{\circ} \mathrm{C}$, the 002 -intensity is already much stronger perpendicular to the fiber and lower parallel to the fiber axis. Perpendicular to the fiber axis for the second ring a hump between 5.5 and $6 \mathrm{~nm}^{-1}$ can be recognized next to the reduced main peak, which is formed by the 101 reflection $\left(4.925 \mathrm{~nm}^{-1} I_{\text {rel }} \approx 18 \%\right.$ ) with little contributions of $100\left(4.694 \mathrm{~nm}^{-1} I_{\text {rel }} \approx 3 \%\right.$ ). This hump can be explained with the 004 peak $\left(5.952 \mathrm{~nm}^{-1}\right)$, which is the second order reflection of 002. Parallel to the fiber axis 004 is less intense and the main peak is dominated by 101 and shows only long decay towards larger scattering vectors. At $800{ }^{\circ} \mathrm{C}$, this behavior becomes even more pronounced. Nevertheless, the texture effect in the two subrings of the second ring in the diffraction patterns in Fig. 7(e) and (f) is much weaker than in the FFTs of the HRTEM micrographs recorded at the side surface of fiber 5 in Fig. $4(\mathrm{~b}-\mathrm{d})$.

During the last heating step up to $1000{ }^{\circ} \mathrm{C}$, the nanofiber selected for diffraction moved on the heating chip, so the orientation of the fiber and the position of the selected area aperture are not identical to the previous measurements at the other temperatures. Nevertheless, a diffraction pattern was recorded and is displayed in Fig. 8 together with the corresponding micrograph as well as with the radial intensity extracted parallel and perpendicular to the fiber axis. According to the different fiber orientation, the angular ranges for extracting the radial intensities parallel and perpendicular to the fiber axis changed to $175-215^{\circ}$ (parallel) and $265-305^{\circ}$ (perpendicular). The texture effects at $1000{ }^{\circ} \mathrm{C}$ are more pronounced compared to the results at lower temperatures, with almost equal intensity of 002 and $100+101$ parallel to the fiber axis. The presence of texture in diffraction arising from a volume close to the center of the fiber means the texture is also present inside the fiber. The observation of diffraction rings up 

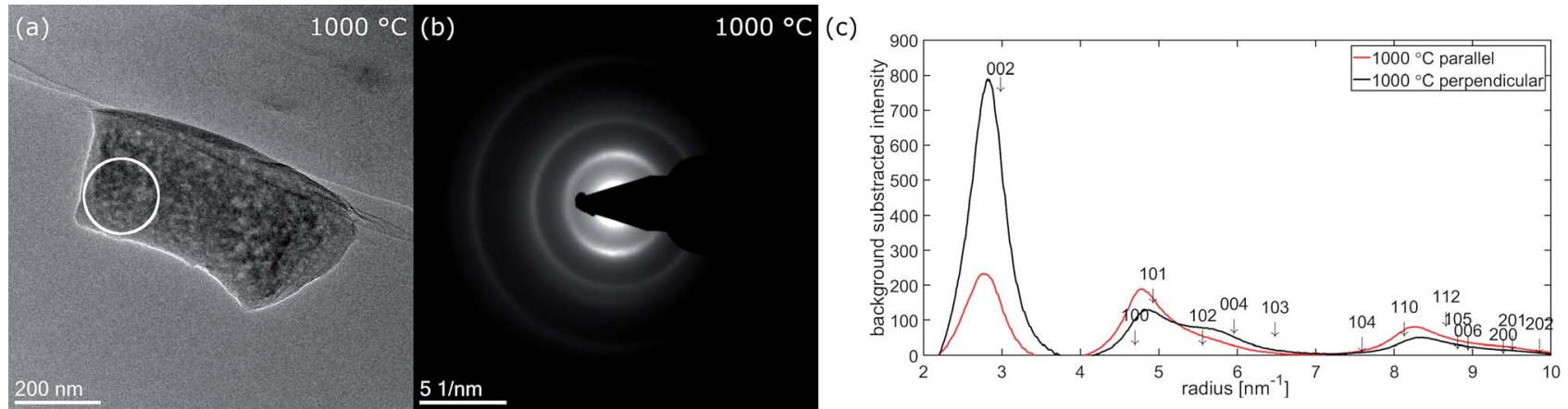

Fig. 8 (a) Fiber 2 in slightly different orientation at $1000{ }^{\circ} \mathrm{C}$ with the position of the SA for diffraction marked by a the white circle (b) electron diffraction pattern at $1000^{\circ} \mathrm{C}$ (c) extracted background substracted radial intensities parallel (red) and perpendicular (black) to the fiber axis subtracted radial intensity parallel and perpendicular to the fiber axis.

to $10 \mathrm{~nm}^{-1}$ correlates with an extended graphitic order. This is supported by Raman spectra measured ex situ on fiber mats carbonized for $10 \mathrm{~h}$ in argon atmosphere at the same temperatures shown in Fig. S3. $\uparrow$ The spectra are scaled to the D-band maximum at around $1350 \mathrm{~cm}^{-1}$. An increase of G-band intensity around $1580 \mathrm{~cm}^{-1}$ with increasing carbonization temperature is clearly visible.

\section{Discussion}

In situ TEM carbonization experiments provide an approach to investigate the morphological and structural changes during carbonization based on observations of individual nanofibers. In combination with spectroscopic methods such as EELS and energy dispersive X-ray spectroscopy (EDX) available in the TEM also the evolution of the chemical composition can be investigated in situ. By tracking the evolution of fiber diameter and structure of the same nanofiber up to temperatures of $1000{ }^{\circ} \mathrm{C}$, statistical scattering, which stems from comparing different fibers in ex situ experiments, can be avoided. In fact, analysis of SEM images (Fig. S1 and S2 $\dagger$ ) resulted in a broad distribution of fiber diameters. In situ TEM enables to measure the shrinkage in diameter on the identical fiber at any time during an experiment. After $1000{ }^{\circ} \mathrm{C}$, we observed shrinkage in the range of 15 to $20 \%$ in diameter, which correlates to a loss in cross sectional area in the range from 27 to $35 \%$. Musiol et al. reported a weight loss of $\approx 39 \%$ for carbon microfibers and $55 \%$ for carbon nanofibers measured by thermogravimetry. ${ }^{18}$ Translating the shrinkage observed in our study into a mass loss, the shrinkage in our experiment is comparable. Differences could arise from different fibers used at the initial state and the formation of porosity, which could increase the mass loss with respect to shrinkage of fiber diameter.

By intermediate TEM magnification micrographs, we observed a marked increase of the nanofiber roughness, especially in the first heating step to $600{ }^{\circ} \mathrm{C}$. The surface roughening was accompanied by diffraction contrast arising inside the fibers. Roughening of carbon nanofibers derived from electrospun PAN upon graphitization at higher temperatures of 1500 and $2800{ }^{\circ} \mathrm{C}$ was also reported by Kurban et al. ${ }^{27}$ Bennett used diffraction contrast in dark-field TEM to image regions of turbostratic graphite in carbon microfibers treated at temperatures. ${ }^{21}$ In our study, we observe regions with sizes around $5 \mathrm{~nm}$ after $600{ }^{\circ} \mathrm{C}$ and up to $10 \mathrm{~nm}$ after $800{ }^{\circ} \mathrm{C}$ or even up to15 $\mathrm{nm}$ at $1000{ }^{\circ} \mathrm{C}$. These sizes agree with our HRTEM micrographs, where we estimate the size of ordered regions within the graphitic plane $\left(L_{10}\right.$ or $\left.L_{\mathrm{a}}\right)$ and perpendicular to them $\left(L_{002}\right.$ or $L_{\mathrm{c}}$ ) of few $\mathrm{nm}$ at $600{ }^{\circ} \mathrm{C}$, up to $5 \mathrm{~nm}$ at $800{ }^{\circ} \mathrm{C}$ and up to $10 \mathrm{~nm}$ at $1000^{\circ} \mathrm{C}$. So the interpretation as diffraction contrast arising from turbostratic carbon is valid. A slightly higher measure in diffraction contrast is not surprising as the field of view and the statistics are better the image resolution poorer. In our case the estimated sizes in and out of plane are comparable. Laffont et al. retrieved $L_{10} \approx 3-4 \mathrm{~nm}$ by Warren Bodenstein formula and $L_{002} \approx 1 \mathrm{~nm}$ retrieved from the Scherrer equation, both from XRD results. ${ }^{23,33} \mathrm{Kim}$ et al. achieves similar results for $L_{\mathrm{c}}$ from XRD via the Scherrer equation. ${ }^{34}$ In their work the values for $L_{10}$ (or $L_{\mathrm{a}}$ ) extracted from Raman spectra via the Knight and White equation are also larger than the values of $L_{002}{ }^{34,35}$ Deviations of sizes measured with different methods can be expected. In addition results are sensitive to experimental conditions and the initial fibers used.

In agreement with literature we observed a preferential alignment of the graphitic planes with the fiber axis. . $^{1,21,22,27,34}$ This texture is confirmed by the FFTs of the HRTEM images as well as the electron diffraction patterns. Comparing our HRTEM micrographs to others the graphitic regions in our experiment appear more disordered with planes more bend and also the borders of the ordered regions are less defined. ${ }^{18,21,22,27,34}$ Most similarity is found with the micrographs published by Kim et al. However clearly in our micrographs a higher number of planes stacked corresponding to higher $L_{\mathrm{c}}$ is visible. $^{20}$ The curvature of graphitic planes observed in HRTEM is well reflected by the rather broad peaks in the azimuthal distribution within the diffraction rings in the FFTs and the electron diffraction patterns. We achieved good agreement of the experimentally observed azimuthal intensity distribution with calculated intensities based on a simple model, which correlates to a variation in orientation of the graphitic (002)planes. A different degree of texture for HRTEM-micrographs recorded at the fiber surface, the broken tip of the nanofiber and electron diffraction was observed. This can be explained 
with the volume probed in each experiment. In the HRTEM micrographs of the fiber surface in Fig. 4(a-d) only the structure of the surface up to a depth of $\approx 30 \mathrm{~nm}$ is probed. With the texture proposed in Fig. 5(a), similar to that suggested by Bennet for microfibers, most of the (002)-planes are parallel to the electron beam in this region of the fiber resulting in enhancement of 002-reflection compared to the averaged structure. ${ }^{11}$ In the electron diffraction experiment on the other hand, the selected area aperture is positioned more central to the fiber axis (Fig. 5(b) and $7(\mathrm{a}-\mathrm{c})$ ) so diffraction arises from the central volume of the fiber plus the upper and lower surface. In this part of the fiber (002)-planes are expected in perpendicular orientation to the electron beam and therefore not in diffraction condition. The aperture with a diameter of $\approx 170 \mu \mathrm{m}$ in the intermediate image plane cuts of regions close to the surface which contribute to the HRTEM-micrographs taken at the fiber surface. Nevertheless texture effects are observed, underlining that, even with some distance to the nanofiber surface, the texture still is present. However, the HRTEM-micrographs recorded at the broken tip of a nanofiber don't show texture. Within the region chosen there, the turbostratic carbon is randomly oriented. We propose to apply both HRTEM and diffraction as these two techniques are complementary in respect to the volume probed. While for the analysis of nanofibers designed for mechanical applications the knowledge about the structures in the fiber cores is crucial, properties of the surfaces and surface near regions are the most relevant for the application of nanofibers in electrodes for metal-air batteries, fuel cells or electrocatalysis.

Quantitative analysis of the diffraction data showed that the 002 peak stabilizes around $2.84 \mathrm{~nm}^{-1}$ at $800{ }^{\circ} \mathrm{C}$ and $1000{ }^{\circ} \mathrm{C}$ corresponding to a $d_{002}$-spacing of $352 \mathrm{pm}$ compared to $335 \mathrm{pm}$ for graphite. ${ }^{25}$ Laffont et al. and Kim et al. reported during stabilization like our fiber mat, after $1000{ }^{\circ} \mathrm{C}$ heat treatment part of the nitrogen remains in the fiber. ${ }^{\mathbf{2 0} 26}$ However, in the case of an in situ TEM experiment an influence of UHV compared to inert gas atmosphere in an ex situ experiment on $d_{002}$-values of $349 \mathrm{pm}$ and $357 \mathrm{pm}$ respectively for PAN derived carbon fibers. ${ }^{23,34}$ Our result is comparable to both of them. Kim et al. attribute the stacking distance to the presence of quaternary nitrogen. ${ }^{20}$ Laffont et al. showed that in fiber set 2 , which was allowed to shrink the nitrogen content is possible. If the microscope is equipped with a spectrometer, the chemical composition can be tracked by EELS or EDX during the in situ experiment.

As shown above the techniques available in a TEM allow a broad spectrum of analysis to study the carbonization of PANderived nanofiber during an in situ experiment. Results are at least comparable to ex situ experiments. However, it has to be kept in mind that a complete reproduction of ex situ experiments inside the TEM is not possible as parameters such as heating rate and atmosphere affect the final properties of the nanofibers. ${ }^{34,36}$ Combined in situ heating experiments under gas flow, which are possible nowadays, could help to circumvent this issue. During the first $250{ }^{\circ} \mathrm{C}$ heating step some influence of the electron beam was evidenced from the substantial difference in fiber diameter shrinkage, depending on the exposure of the nanofiber to the electron beam. The latter might be attributed to the interaction of the electron beam with residual gases in the microscope and the applied heating of the sample. ${ }^{37-39}$ The fibers with higher exposure to the electron beam under UHV conditions at $250{ }^{\circ} \mathrm{C}$ show faster shrinkage. Also an influence on the surface roughness is possible. This could be controlled by a comparison with additional ex situ experiments on identical locations. In any case, the beam exposure should be kept to a minimum, which we did for the rest of the fibers. With respect to the main subject of research - the formation of graphitic structures in the fibers depending on carbonization temperature - this confinement does not preclude the in situ method for these objectives.

With the combination of TEM at low, intermediate and high magnification and electron diffraction we were able to follow the shrinkage of fiber diameter and the development of the surface morphology as well as the carbon structure during the in situ experiment. In combination with complementary ex situ experiments, the in situ experiments can contribute to an overall picture of the carbonization process for polymer-derived carbon nanofibers. Thus, the in situ carbonization TEM technique provides a promising approach which is currently just at the beginning of its development.

\section{Conclusions}

Dimensional changes, surface morphology as well as the structure of PAN-derived carbon nanofibers were investigated by in situ TEM during their carbonization up to $1000{ }^{\circ} \mathrm{C}$. Shrinkage in diameter as observed on individual nanofibers over the whole temperature range mounts up to $20 \%$ reduction of the initial size. No marked influence of the initial nanofiber diameter on its relative reduction was detected. Enhanced shrinkage - in particular at the $250{ }^{\circ} \mathrm{C}$ temperature stage - was indicated for nanofibers, which were subject to intensive exposure to the electron beam. As a second major result, intermediate resolution TEM imaging clearly revealed the roughening of the surface, which is beneficial for catalytic applications. Moreover, intermediate TEM also revealed the transition from amorphous contrast to an increased diffraction contrast on the length scale of about $5 \mathrm{~nm}$ at $600{ }^{\circ} \mathrm{C}$ to $\approx 10 \mathrm{~nm}$ at $800{ }^{\circ} \mathrm{C}$ and even up to $15 \mathrm{~nm}$ at $1000{ }^{\circ} \mathrm{C}$. HRTEM showed a similar behavior with slightly smaller sizes. Furthermore, HRTEM and its FFT showed the presence of turbostratic ordered regions for temperatures $600{ }^{\circ} \mathrm{C}$ and above, with preferential alignment of the (002)-planes parallel to the fiber axis on the lateral surfaces. The more graphitic structure should increase the electronic conductivity of the nanofibers for electrochemical applications. Finally, electron diffraction showed that the same texture continues inside the nanofiber. The observation of higher order reflections confirms the growth of the ordered regions. Based on quantitative evaluation of the 002-ring in the electron diffraction patterns a $d_{002}$ spacing of $\approx 352$ pm was determined at the end of the experiment. Overall, in situ TEM, with its possibility of different imaging techniques, diffraction and also spectroscopic methods provides a powerful tool to study the 
carbonization of PAN-derived nanofibers on identical locations at any time during the experiment.

\section{Conflicts of interest}

There are no conflicts to declare.

\section{Acknowledgements}

The work has been supported by the Bundesministerium für Bildung und Forschung (BMBF) under the Project LuZi (Förderkennzeichen 03SF0499F). The dual beam machine FEI Helios 460 F1 was funded by the Bundesministerium für Bildung und Forschung (BMBF) under the Project "SABLE", Förderkennzeichen 03EK3543.

\section{References}

1 X. Lu, C. Wang and Y. Wei, Small, 2009, 5, 2349-2370.

2 A. M. Al-Enizi, A. A. Elzatahry, A. M. Abdullah, A. Vinu, H. Iwai and S. S. Al-Deyab, Appl. Surf. Sci., 2017, 401, 306-313.

3 J. Chen, J. Chen, D. Yu, M. Zhang, H. Zhu and M. Du, Electrochim. Acta, 2017, 246, 17-26.

4 M. Li, G. Han and B. Yang, Electrochem. Commun., 2008, 10, 880-883.

5 K. Gong, F. Du, Z. Xia, M. Durstock and L. Dai, Science, 2009, 323, 760 .

6 L. Huang, Q. Guan, J. Cheng, C. Li, W. Ni, Z. Wang, Y. Zhang and B. Wang, Chem. Eng. J., 2018, 334, 682-690.

7 H. Wang, J. Deng, C. Xu, Y. Chen, F. Xu, J. Wang and Y. Wang, Energy Storage Materials, 2017, 7, 216-221.

8 J. Zhu, L. Chen, Z. Xu and B. Lu, Nano Energy, 2015, 12, 339346.

9 A. Greiner and J. H. Wendorff, Angew. Chem., 2007, 119, 5770-5805.

10 S. Damodaran, P. Desai and S. Abhiraman A, J. Text. Inst., 1990, 81, 384-420.

11 P. J. Goodhew, A. J. Clarke and J. E. Bailey, Mater. Sci. Eng., 1975, 17, 3-30.

12 M. Balasubramanian, M. K. Jain, S. K. Bhattacharya and A. S. Abhiraman, J. Mater. Sci., 1987, 22, 3864-3872.

13 W. N. Turner and F. C. Johnson, J. Appl. Polym. Sci., 1969, 13, 2073-2084.

14 M.-S. Balogun, W. Qiu, F. Lyu, Y. Luo, H. Meng, J. Li, W. Mai, L. Mai and Y. Tong, Nano Energy, 2016, 26, 446-455.

15 E. S. Davydova, A. Y. Rychagov, I. I. Ponomarev and I. I. Ponomarev, Russ. J. Electrochem., 2013, 49, 1010-1011.

16 N. Grassie and R. McGuchan, Eur. Polym. J., 1970, 6, 12771291.
17 K. Molnár, B. Szolnoki, A. Toldy and L. M. Vas, J. Therm. Anal. Calorim., 2014, 117, 1123-1135.

18 P. Musiol, P. Szatkowski, M. Gubernat, A. WeseluchaBirczynska and S. Blazewicz, Ceram. Int., 2016, 42, 1160311610.

19 R. Ojeda-López, G. Ramos-Sánchez, J. M. Esparza-Schulz, L. Lartundo and A. Domínguez-Ortiz, Int. J. Hydrogen Energy, 2017, 42, 30339-30348.

20 S.-W. Park, J.-C. Kim, M. A. Dar, H.-W. Shim and D.-W. Kim, Chem. Eng. J., 2017, 315, 1-9.

21 S. C. Bennett, PhD thesis, University of Leeds, 1976.

22 Y.-J. Bai, C.-G. Wang, N. Lun, Y.-X. Wang, M.-J. Yu and B. Zhu, Carbon, 2006, 44, 1773-1778.

23 L. Laffont, M. Monthioux and V. Serin, Carbon, 2002, 40, 767-780.

24 P. H. Gamlen and J. W. White, J. Chem. Soc., Faraday Trans. 2, 1976, 72, 446-455.

25 P. Trucano and R. Chen, Nature, 1975, 258, 136.

26 L. Laffont, M. Monthioux, V. Serin, R. B. Mathur, C. Guimon and M. F. Guimon, Carbon, 2004, 42, 2485-2494.

27 Z. Kurban, A. Lovell, D. Jenkins, S. Bennington, I. Loader, A. Schober and N. Skipper, Eur. Polym. J., 2010, 46, 11941202.

28 S. Prilutsky, E. Zussman and Y. Cohen, J. Polym. Sci., Part B: Polym. Phys., 2010, 48, 2121-2128.

29 L. F. Allard, W. C. Bigelow, M. Jose-Yacaman, D. P. Nackashi, J. Damiano and S. E. Mick, Microsc. Res. Tech., 2009, 72, 208215.

30 M. Kruth, D. Meertens and K. Tillmann, Journal of LargeScale Research Facilities, 2016, 2, A59.

31 A. Thust, J. Barthel and K. Tillmann, Journal of Large-Scale Research Facilities, 2016, 2 , A41.

32 K. W. Urban, C.-L. Jia, L. Houben, M. Lentzen, S.-B. Mi and K. Tillmann, Philos. Trans. R. Soc., A, 2009, 367, 3735.

33 B. E. Warren and P. Bodenstein, Acta Crystallogr., 1966, 20, 602-605.

34 M.-A. Kim, D. Jang, S. Tejima, R. Cruz-Silva, H.-I. Joh, H. C. Kim, S. Lee and M. Endo, Sci. Rep., 2016, 6, 22988.

35 D. S. Knight and W. B. White, J. Mater. Res., 2011, 4, 385-393. 36 J. Cao, W. Zhao and S. Gao, Materials, 2018, 11.

37 A. L. Koh, E. Gidcumb, O. Zhou and R. Sinclair, ACS Nano, 2013, 7, 2566-2572.

38 A. L. Koh, E. Gidcumb, O. Zhou and R. Sinclair, Nano Lett., 2016, 16, 856-863.

39 A. L. Koh and R. Sinclair, Ultramicroscopy, 2017, 176, 132138. 